\section{Near-infrared reflectance and autofluorescence imaging characteristics of choroidal nevi}

NA Vallabh ${ }^{1,2}, J_{N}$ Sahni ${ }^{1,2}$, CK Parkes $^{2}$, G Czanner ${ }^{1}$, H Heimann ${ }^{1,2}$ and B Damato ${ }^{1,3}$

\begin{abstract}
Purpose To report near-infrared reflectance (NIR-R), near-infrared autofluorescence (NIR-AF) and blue wave autofluorescence (BW-AF) appearance of choroidal nevi using a confocal scanning laser ophthalmoscope (cSLO).

Patients and methods NIR-R, NIR-AF and BW-AF images of choroidal nevi were compared with color fundus photos (CF). Images were graded as hyperreflective if reflectance was much greater than background, hyporeflective if less than background, and isoreflective if the same as the background.

Results Forty-two nevi of 39 patients were
\end{abstract} imaged. When compared with $C F$, nevi could be identified on $95 \%(40 / 42)$ NIR-R images (95\% CI: 83.5-99.3). On NIR-R 71\% (30/42) demonstrated hyperreflectance and $24 \%$ (10/42) were hyporeflective. Hyperreflectivity was demonstrated in $96 \%(23 / 24)$ of NIR-AF images (95\% CI: 79.1-99.9) and 34\% (14/41) of BW-AF images (95\% CI: 20.0-50.5). On NIR-R, $29 / 40(73 \%)$ were apparently smaller in comparison with CF and $11 / 40(28 \%)$ had the same area. A correlation was found between NIR-R and NIR-AF $(P=0.02)$ but not with BW-AF $(P=0.15)$.

Conclusions Nevi can be visualized well using NIR-R and NIR-AF imaging modalities, but are less frequently visible using BW-AF. These changes may be related to melanin within the choroid or chronic changes of the overlying retinal pigment epithelium.

Eye (2016) 30, 1593-1597; doi:10.1038/eye.2016.183; published online 2 September 2016

\section{Introduction}

The prevalence of choroidal nevi in the Caucasian population in the United States has been estimated to be between 4.6 and $7.9 \%{ }^{1}$
The annual rate of malignant transformation of a choroidal nevus was estimated to be 1 in $8845 .{ }^{1}$ Monitoring choroidal nevi for progression is done primarily using color fundus photography (CF), with or without B-scan ultrasonography. In recent times, optical coherence tomography (OCT) and blue wave autofluorescence $488 \mathrm{~nm}$ (BW-AF) have provided additional information in suspicious lesions.

Presence of BW-AF, signifying lipofuscin, may herald early transformation of a melanocytic nevus to melanoma. ${ }^{2}$ However, with $488 \mathrm{~nm}$ $\mathrm{BW}-\mathrm{AF}$ there is little penetration of light beyond the retinal pigment epithelium (RPE). Studies by Lavinsky et al, ${ }^{3}$ Keilhauer and Delori ${ }^{4}$ reported normal patterns of background fundus fluorescence on autofluorescence of choroidal nevi, hence they are not visible on BW-AF imaging.

Although papers have reported near-infrared autofluorescence $787 \mathrm{~nm}$ (NIR-AF) and nearinfrared reflectance $815 \mathrm{~nm}$ (NIR-R) in the normal and abnormal retina, there is little information regarding near-infrared imaging characteristics of choroidal nevi. ${ }^{5-8}$ In this study we describe the NIR-R, NIR-AF and BW-AF characteristics of choroidal nevi, using confocal scanning laser ophthalmoscope (CSLO), and how these findings correlate to one another.

\section{Materials and methods}

Consecutive patients undergoing fundus imaging, as part of their routine assessment for monitoring choroidal nevi, were included. This study had the approval of the Clinical Governance department (Number AC02649).

The diagnosis of a nevus was confirmed by a lack of risk factors for melanoma. These lesions demonstrated no elevation clinically. OCT was performed to ensure no elevation, no subretinal fluid and that all lesions were $<5 \mathrm{~mm}$ in
${ }^{1}$ Department of Eye and Vision Science, Institute of Ageing and Chronic Disease, Faculty of Health and Life Sciences, University of Liverpool, Liverpool, UK

${ }^{2}$ St Paul's Eye Unit, Royal Liverpool University Hospital, Liverpool, UK

${ }^{3}$ University of California, San Francisco, CA, USA

Correspondence: NA Vallabh, St Paul's Eye Unit, Royal Liverpool University Hospital, Prescot Street, Liverpool L7 8XP, UK Tel: +44 (0)151 7062000. E-mail: neeruvallabh@ hotmail.co.uk

Received: 28 September 2015

Accepted in revised form: 28 June 2016

Published online:

2 September 2016 
diameter on OCT. Some patients had the presence of one risk factor for melanoma and this was also recorded, which included lesions $<3 \mathrm{~mm}$ from the optic disc or lipofuscin.

All patients were imaged with a Topcon Color fundus camera (Topcon TRC.50EX, Tokyo, Japan) and Heidelberg Spectralis SLO (Heildelberg Spectralis, Heidelberg Engineering, Dossenheim, Germany) for BW-AF, NIR-R, NIR-AF, and OCT.

Infrared imaging was performed using the $30^{\circ}$ or $55^{\circ}$ field of view and $1536 \times 1536$ pixel resolution. NIR-R images were acquired using an $815 \mathrm{~nm}$ diode laser. NIR-AF images were acquired by using a diode laser of $787 \mathrm{~nm}$ for excitation and a barrier filter allowing return light passage $>810 \mathrm{~nm}$ (near-infrared spectrum). BW-AF images were acquired using $488 \mathrm{~nm}$ for excitation and a barrier filter $>500 \mathrm{~nm}$. Images were averaged for correction of eye movements using the Heidelberg automatic real time tracking software. Spectral domain OCT (SD-OCT) and Enhanced Depth Imaging OCT (OCT-EDI) images were taken using Spectralis Heildelberg Spectralis (Heidelberg Engineering, Dossenheim, Germany).

\section{Grading of retinal images}

Images were analyzed and graded independently by two ophthalmic specialist trainees (NV and $\mathrm{CP}$ ). They underwent further assessment by a senior ophthalmologist consultant JS, particularly when discrepancies were found. CF were graded for quality, pigmentation and margins. CF quality was graded as good, fair or poor based on the criteria developed by NetwORC UK for the IVAN Study. ${ }^{9}$ These principles were also applied for the use in the IVAN trial. ${ }^{9}$ Presence of drusen and/or lipofuscin were also noted.

Heidelberg SLO images were graded for reflectance and autofluorescence properties and compared with color fundus photos. NIR-R, NIR-AF and BW-AF images were graded as hyperreflective if the reflectivity was greater

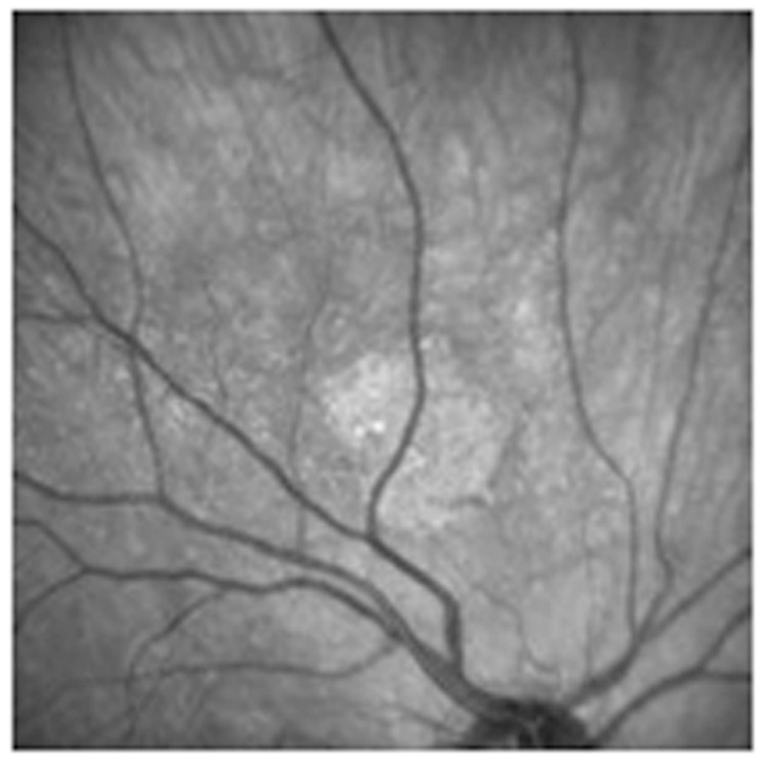

Figure 1 Standard NIR-R image. Reference photograph to mark the boundary between hyperreflectivity and isoreflectivity.
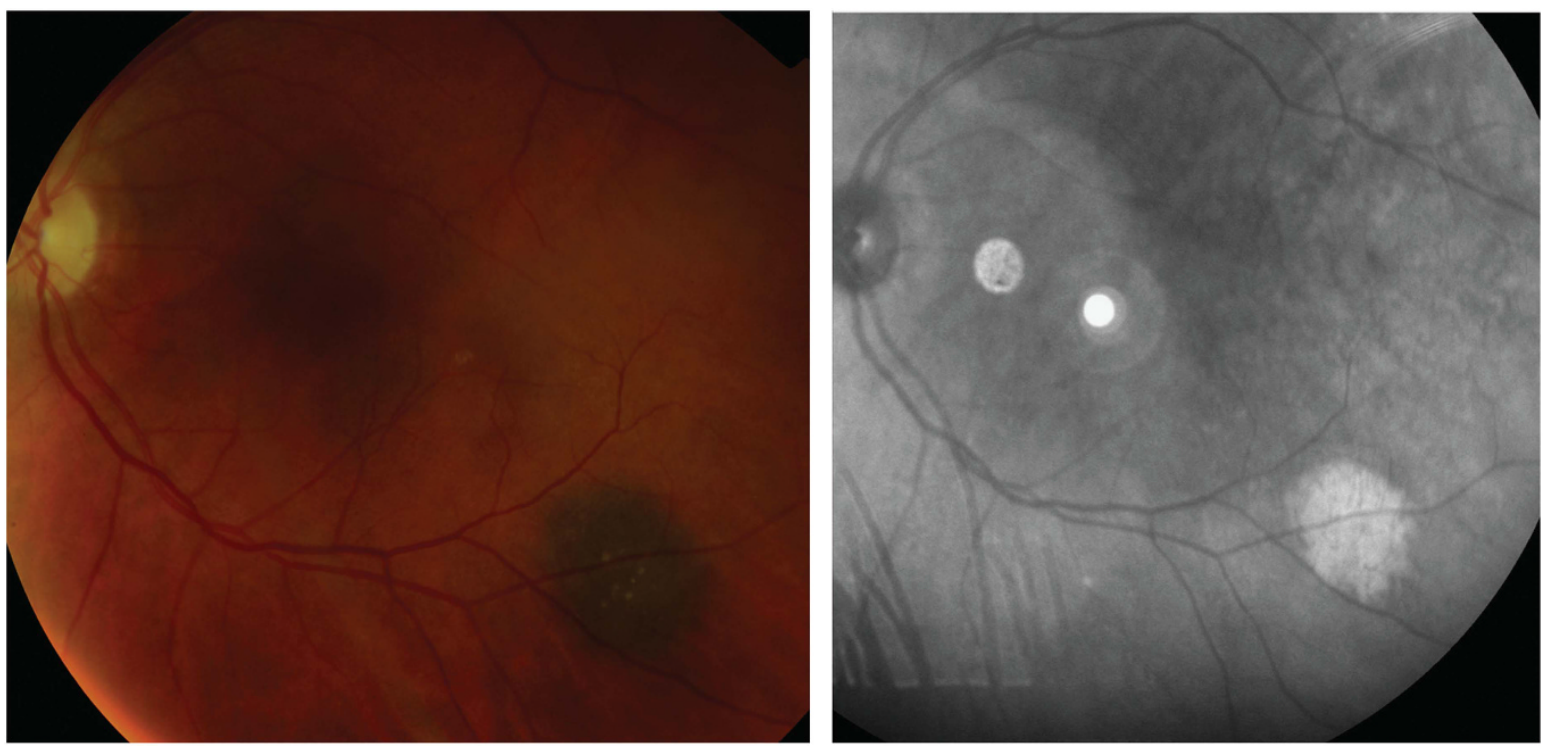

Figure 2 An illustration of a choroidal nevus in the left eye of a patient exhibiting hyperreflectivity- Left: Colour fundus photo (CF) image, Right: NIR-R of the same patient. 
than background and hyporeflective if less than background. Figure 1 demonstrates NIR-R imaging criteria. If the nevus was not visible it was deemed to exhibit isoreflectivity. The area of the nevus on CF was compared with the area of hyperreflectivity using delineation of the lesions according to the local landmarks.

SD-OCT was reviewed for the presence of subretinal fluid (SRF) and pigment epithelial defects (PED).

The data was analyzed using Testing for one proportion analysis and calculation of $95 \%$ confidence intervals of the data. ${ }^{10} \mathrm{~A}$ null hypothesis was determined prior to the study that choroidal nevi would not be visible in over $80 \%$ of cases. This allowed for $P$ value calculation using the test for one proportion. Pearson's $\chi 2$-testing was used to analyze correlation between differing modalities.

\section{Results}

Forty-six consecutive patients (20 male; 26 female; average age 66.5 years (27-89 years)) underwent CF, OCT, NIR-R, NIR-AF and BW-AF imaging. Seven sets of images were excluded due to poor digital color fundus image quality. Table 1 is a summary of the number of images available.

Sixteen of the 42 (38\%) had overlying drusen and 3/42 $(6 \%)$ had lipofuscin. None of the nevi demonstrated SRF or PEDs on OCT.

\section{Near-infrared reflectance}

- Nevi were visible in 40 of 42 NIR-R images (95\%; P $=0.015$, 95\% CI: 83.5-99.3)

- Two (5\%) were isoreflective (not visible)

- 30/42 (71\%) were hyperreflective (see Figure 2 as an example) and 10/42 (24\%) were hyporeflective compared with the background

- $21 / 40(53 \%)$ appeared smaller in size on NIR-R compared with CF (Table 2).

\section{Near-infrared autofluorescence}

- Nevi were visible in 23 of the 24 NIR-AF images (96\%; P $=0.050$, 95\% CI: 79.1-99.9)

- $1 / 24(4 \%)$ was isoreflective (not visible)

- 23/24 were hyperreflective (96\%), none were hyporeflective.

- $10 / 24(42 \%)$ appeared smaller in size on NIR-AF compared with CF.

\section{Blue wave autofluorescence}

- Nevi were visible in $14 / 41$ (34\%; $P<0.0001,95 \%$ CI: 20.0-50.5)

- $27 / 41(66 \%)$ was isoreflective (not visible)

- $13 / 41(32 \%)$ were hyperreflective, none were hyporeflective.

- All 13 appeared smaller in size on BW-AF compared with CF.

\section{Correlation between imaging modalities}

Pearson's $\chi 2$-test was performed to analyze if hyperreflectivity and iso-/hyporeflectivity on NIR-R correlated with the visibility on NIR-AF. A relationship was demonstrated between level of NIR-R reflectivity and nevi visibility on NIR-AF, $X^{2}(1, n=24)=5.2174, P=0.02$. Pearson $\chi 2$-test demonstrated no correlation between level of NIR-R reflectivity and nevi visibility on BW-AF, $X^{2}(1, n=41)=2.0438, P=0.15$. There was no correlation between visibility on NIR-AF and BW-AF $X^{2}$ $(1, n=24)=0.209, P=0.65$.

\section{Impact of risk factors on imaging modality findings}

Lesions were analyzed for risk factors for melanoma, to see the impact of these on imaging characteristics. Interestingly although all three lesions with lipofuscin showed increased BW-AF, two demonstrated hyperreflectivity on NIR-R and one demonstrated hyporeflectivity. There were no images with lipofuscin that had good quality images for NIR-AF. Six lesions were juxtapapillary in location $(<3 \mathrm{~mm})$ and these all

Table 1 Retinal images available for analysis

\begin{tabular}{lccc}
\hline $\mathrm{N}=42$ & NIR-R & NIR-AF & $B W-A F$ \\
\hline Good quality image & 42 & 24 & 41 \\
Could not grade due to poor & 0 & 16 & 0 \\
image quality & & 2 & 1 \\
Imaging modality not performed & 0 & 2 & \\
\hline
\end{tabular}

Table 2 Level of reflectivity on differing imaging modalities

\begin{tabular}{lccc}
\hline & \multicolumn{3}{c}{ Levels of Reflectivity } \\
\cline { 2 - 4 } & Isoreflectivity & Hyporeflectivity & Hyperreflectivity \\
\hline NIR-R & $2 / 42(5 \%)$ & $10 / 42(24 \%)$ & $30 / 42(71 \%)$ \\
NIR-AF & $1 / 24(4 \%)$ & & $23 / 24(96 \%)$ \\
BW-AF & $27 / 41(66 \%)$ & & $14 / 41(34 \%)$ \\
\hline
\end{tabular}


demonstrated hyperreflectivity on NIR-R. Two had images of NIR-AF not suitable for grading but the remaining four showed increased NIR-AF.

\section{Discussion}

In our case series, the presence of NIR-R hyperreflectivity within choroidal nevi was a common finding (71\%). Interestingly in some instances the area of increased reflectivity was smaller in size when compared with the size on CF in 21/40 (53\%). NIR-AF images also demonstrated increased reflectivity in $96 \%$ of cases, but this imaging modality proved difficult to obtain, with only 24 out of 40 images being suitable enough to grade. Increased BW-AF (488 nm) was visible over nevi in 14 of the 41 available images (34\%). This corresponded to autofluorescence (AF) from drusen overlying the nevi in six cases and lipofuscin in three patients. NIR-R correlated with visibility on NIR-AF $\left(X^{2}(1, n=24)=5.2174 P=0.02\right)$, however no correlation was demonstrated between BW$\mathrm{AF}$ and the other imaging modalities.

A study by Weinberger et $a l^{8}$ examining different imaging modalities in patients with a variety of macular diseases reported increased NIR-R and NIR-AF in 4 of 6 $(67 \%)$ patients with choroidal nevi ( $c f .71 \%$ of 42 patients in our cohort). Lavinsky et al analyzed BW-AF in 15 nevi. They found that two out of 15 (13\%) nevi imaged with this technique demonstrated hyperautofluorescence corresponding to drusen. ${ }^{3}$ This is similar to our cohort of patients. Keilhauer and Delori ${ }^{4}$ found no difference in BW-AF of nevi as compared with normal background fundus. A paper by Amselem et al ${ }^{11}$ analyzed infrared reflectance (IRR) and compared these with BW-AF in seven patients with choroidal melanomas. They found a correlation between detection of lipofuscin in both imaging modalities, but that IRR was found to be inferior to BW-AF. ${ }^{11}$ This is interesting as in our cohort we found increased levels of NIR-R in nevi compared with BW-AF and there was no statistical correlation found between the two imaging modalities.

It has been reported that malignant melanocytic cells in the choroid have a direct effect on the overlying retinal pigment epithelium. ${ }^{12}$ This epitheliopathy causes areas of atrophy, subretinal fluid, and accumulation of lipofuscin, melanolipofuscin and pigment laden macrophages. ${ }^{12}$ These changes to the RPE produces fluorophores which, on excitation with blue wave light, exhibit fundus AF. It has been proposed that melanin (in the RPE and choroid) is responsible for the increase in both NIR-R and NIR-AF. ${ }^{4,6-8}$

A longitudinal study is needed to ascertain if there are any changes in NIR-R and NIR-AF within nevi over time, in the rare instances when nevi change into melanomas. The disadvantage of this study is the lack of tissue specimens to analyze due to the benign nature of this condition. The clinical use of these imaging modalities in nevi monitoring, imaging and potential malignant transformation need to be explored further.

\section{Conclusion}

In summary, to our knowledge, this is the first report on the baseline NIR characteristics of a larger cohort of patients with choroidal nevi. It has been demonstrated that NIR-R and NIR-AF were increased in choroidal nevi. Statistically the visibility of these lesions were found to correlate between these two imaging modalities. The presence of NIR-R and NIR-AF did not correlate with BW-AF findings. Further research is required to see if this may assist in early differentiation of melanoma from nevi, particularly in cases of indeterminate lesions.

\section{Summary}

\section{What was known before}

- Previous groups have found increased near-infrared reflectance (NIR-R) and near-infrared autofluorescence (NIR-AF) in choroidal nevi in a small number of patients $(n=6)$.

- Blue wave autofluorescence (BW-AF) features of nevi have been previously described.

\section{What this study adds}

- First report on baseline NIR-R and NIR-AF characteristics in a larger cohort of choroidal nevi $(n=42)$.

- This study correlates findings between BW- AF, NIR-R and NIR-AF.

\section{Conflict of interest}

The authors declare no conflict of interest.

\section{References}

1 Singh AD, Kalyani P, Topham A. Estimating the risk of malignant transformation of a choroidal nevus. Ophthalmology 2005; 112(10): 1784-1789.

2 Piccolino FC, Borgia L, Zinicola E, Iester M, Torrielli S. Pre-injection fluorescence in indocyanine green angiography. Ophthalmology 1996; 103: 1837-1845.

3 Lavinsky D, Belfort RN, Navajas E, Torres V, Martins MC, Belfort R. Fundus autofluorescence of choroidal nevus and melanoma. Br J Ophthalmol 2007; 91 : 1299-1302.

4 Keilhauer CN, Delori FC. Near-infrared autofluorescence imaging of the fundus: visualisation of Ocular Melanin. Invest Ophthalmol Vis Sci 2006; 47: 3556-3564.

5 Ashikari M, Ozeki H, Tomida K, Sakurai E, Tamai K, Ogura Y. Retention of dye after indocyanine green-assisted internal 
limiting membrane peeling. Am J Ophthalmol 2003; 136: 172-174.

6 Elsner AE, Burns SA, Weiter JJ, Delori FC. Infrared imaging of sub-retinal structures in the human ocular fundus. Vision Res 1996; 36: 191-205.

7 Sautter H, Lullwitz W, Naumann G. Infra red photography in the differential diagnosis of suspicious pigmented fundus changes. Klin Monbl Augenheilkd 1974; 164: 597-602.

8 Weinberger AWA, Lappas A, Kirschkamp T, Mazinani BAE, Huth JK, Mohammadi B et al. Fundus near infrared fluorescence correlates with fundus near infrared reflectance. Invest Ophthalmol Vis Sci 2006; 47(7): 3098-3108.
9 A randomised controlled trial of alternative treatments to Inhibit VEGF in Age-related choroidal Neovascularisation (IVAN). IVAN Study- Grading Protocol. Version 1.1. Ophthalmic Research Centre. (Accessed on 20 June 2008).

10 Altman DG. Practical Statistics for Medical Research. London: Chapman and Hall, 1991.

11 Amselem L, Pulido JS, Gunduz K, Garcia-Arumi J, Mones J. Infrared reflectance in choroidal melanoma and its correlation with fundus autofluorescence. $\mathrm{Br} J$ Ophthalmol 2008; 92: 1565-1566.

12 Damato BE, Foulds WS. Tumor-associated retinal pigment epitheliopathy. Eye 1990; 4: 382-387. 